Fields Institute Communications

Volume 00, 0000

\title{
Symplectic Gluing and Family Gromov-Witten Invariants
}

\author{
Junho Lee and Thomas H. Parker \\ Michigan State University \\ East Lansing, MI 48824
}

\begin{abstract}
This article describes the use of symplectic cut-and-paste methods to compute Gromov-Witten invariants. Our focus is on recent advances extending these methods to Kähler surfaces with geometric genus $p_{g}>0$, for which the usual GW invariants vanish for most homology classes. This involves extending the Splitting Formula and the Symplectic Sum Formula to the family GW invariants introduced by the first author. We present applications the invariants of elliptic surfaces and to the Yau-Zaslow Conjecture. In both cases the results agree with the conjectures of algebraic geometers and yield a proof (to appear [LL]) of previously unproved cases of the Yau-Zaslow Conjecture.
\end{abstract}

Gromov-Witten invariants are counts of holomorphic curves in a symplectic manifold $X$. To define them one chooses an almost complex structure $J$ compatible with the symplectic structure and considers the set of maps $f: \Sigma \rightarrow X$ from Riemann surfaces $\Sigma$ which satisfy the (nonlinear elliptic) $J$-holomorphic map equation

$$
\bar{\partial}_{J} f=0 .
$$

After compactifying the moduli space of such maps, one imposes constraints, counting, for example, those maps whose images pass through specified points. With the right number of constraints and a generic perturbation of the equation, the number of such maps is finite. That number is a GW invariant of the symplectic manifold $X$.

The first part of this article focuses on cut-and-paste methods for computing GW invariants of symplectic four-manifolds. Two useful techniques are described in Sections 2-4. The first is the method of "splitting the domain", in which one considers maps whose domains are pinched Riemann surfaces. This produces recursion relations, called TRR formulas, relating one GW invariant to invariants whose domains have smaller area or lower genus.

The second author was partially supported by the N.S.F..

(C)0000 American Mathematical Society 
One can also consider the behavior of GW invariants as one "splits the target" using the symplectic cut operation and its inverse, the symplectic sum. The resulting "Symplectic Sum Formula" of E. Ionel and the second author expresses the GW invariants of the symplectic sum of two manifolds $X$ and $Y$ in terms of the relative GW invariants of $X$ and $Y$ as described in Section 4.

These splitting formulas for the domain and the target can sometimes be combined to completely determine a set of GW invariants. One such computation is given in Section 5. There we use a clever geometric argument of E. Ionel to derive an explicit formula for certain GW invariants of the rational elliptic surface $E(1)$. That formula, explained in Section 5, is

$$
F(t)=\prod_{d \geq 1}\left(\frac{1}{1-t^{d}}\right)^{12}
$$

There is strong motivation for generalizing this $E(1)$ computation. We would, of course, like to make such cut-and-paste arguments into a systematic way of computing invariants of whole classes of 4-manifolds. More alluring yet is the connection with enumerative algebraic geometry. There is a series of conjectures, originally coming from string theory, that claim that the generating functions of counts of holomorphic curves in algebraic surfaces are given by certain specific formulas (see $[G \ddot{o}]$ ). The simplest such formula is exactly $(0.2)$. The next simplest is the Yau-Zaslow conjecture for rational curves in K3 surfaces ([YZ]).

In seeking to generalize the $E(1)$ computation, one encounters a serious problem. For $E(1)$, the GW invariants are the same as the curve counts of enumerative geometry. But for most other complex surfaces, beginning with $K 3$, the conjectural formulas count curves in classes for which the Gromov-Witten invariants vanish!

This discrepancy occurs because GW invariants are defined using generic almost complex structures $J$, while Kähler structures are very special. Entire families of curves can disappear when the Kähler $J$ is perturbed to a generic $J$. For example, algebraic K3 surfaces contain many holomorphic curves, as predicted by the YauZaslow conjecture, but a generic $K 3$ surface has no holomorphic curves at all! In general, given a space (generalized Severi variety) of curves in a Kähler manifold, one can perturb to a generic almost complex structure $J$ and ask which curves in the Severi variety perturb to become $J$-holomorphic curves. This problem fits into standard deformation theory, as explained in Section 6, and from that perspective one sees that there is an obstruction bundle over the Severi variety whose rank is the geometric genus $p_{g}=\operatorname{dim} H^{0,2}(X)$ of the surface $X$. Thus when $p_{g}>0$ the space of curves and the GW moduli space have different dimensions, and consequently the GW invariants are unrelated to the enumerative counts of algebraic geometry.

Sections 7-10 describe the first author's geometric analysis method for overcoming this problem. The key observation is that on each Kähler manifold there is a very natural family of almost complex structures $J_{\alpha}$ parameterized by $H^{0,2}(X)$. One can then consider pairs $(f, \alpha)$ where $f$ is a $J_{\alpha}$-holomorphic map for some $\alpha \in H^{0,2}(X)$. Such pairs form a moduli space whose dimension is $p_{g}$ greater than the standard GW moduli space. The family GW invariants are defined from that moduli space using the constructions of Li-Tian [LT]. (Bryan-Leung and BehrendFantechi, have alternative approaches which use algebraic geometry).

The family moduli space has a remarkable property: whenever $J$ is Kähler, all $J_{\alpha}$-holomorphic maps in fact have $\alpha=0$. Thus the family invariants, which 
are generally counts of $J_{\alpha}$-holomorphic maps, reduce to counts of true holomorphic maps in the Kähler surface. A less pleasing aspect is that the moduli space may not be compact, since $\alpha$ ranges over the vector space $H^{0,2}(X)$. However, results in [L1] show that it is compact in many cases of interest, and for those cases the family invariants are well-defined symplectic invariants that are closely related to enumerative counts.

The next task is to extend the cut-and-paste formulas to the family GW invariants. In Sections 8 and 9 we develop TRR and symplectic sum formulas for the family GW invariants, following [L2]. Those formulas immediately allow us to extend the $E(1)$ calculation of Section 5 to the elliptic surfaces $E(n)$. The result is a simple expression for the family GW invariants which generalizes (0.2).

The final section describes the very recent work of the first author and N.C. Leung on the Yau-Zaslow conjecture. The conjecture predicts a specific formula - a slight modification of $(0.2)$ - for the generating function of the counts of rational curves in K3 surfaces. In [BL1], Bryan and Leung proved the conjecture in the cases when the curves represent primitive homology classes. Sections 9 and 10 describe an independent proof of that fact and its extension to classes that are twice a primitive class. The proofs repeatedly use the symplectic cut-and-paste techniques developed in earlier sections.

\section{Gromov-Witten Invariants}

Fix a closed symplectic four-manifold $(X, \omega)$. Building on ideas of Donaldson and Gromov, one can define symplectic invariants by introducing an almost complex structure $J$ and counting (with orientation) the number of $J$-holomorphic maps into $X$ satisfying certain constraints. This section gives an overview of the setup.

Given $(X, \omega)$, one can always choose an almost complex structure $J$ compatible with $\omega$, i.e. a bundle map $J: T X \rightarrow T X$ with $J^{2}=-$ Id and so that, for tangent vectors $u$ and $v, g(u, v)=\omega(u, J v)$ is a Riemannian metric. Let $\mathcal{J}$ be the space of such $J$. For $J \in \mathcal{J}$, a map $f: C \rightarrow X$ from a complex curve $C$ is $J$-holomorphic if it satisfies

$$
\bar{\partial}_{J} f=0
$$

where $\bar{\partial}_{J} f=\frac{1}{2}(d f+J \circ d f \circ j)$. The $J$-holomorphic maps from a smooth connected domain $C$ with (distinct) marked points $x_{1}, \ldots, x_{k}$ gives a space

$$
\mathcal{M}_{g, k}(X, A)
$$

labeled by the genus $g$ of $C$, the number $k$ of marked points, and the class $A \in$ $H_{2}(X)$ that the image represents.

The space (1.2) has a natural compactification, which can be built in two steps. First, when $X$ is a single point and $k \geq 3-2 g,(1.2)$ is the Deligne-Mumford space of curves. That has a compactification

$$
\overline{\mathcal{M}}_{g, k}
$$

consisting of stable connected curves with arithmetic genus $g$ and $k$ marked points. (A curve is stable if (i) it has only nodal singular points, none of which are marked points, and (ii) each irreducible component of genus $g_{i}$ has at least $3-2 g_{i}$ marked 
or nodal points.) The Deligne-Mumford compactification is an orbifold, and is a complex projective variety of dimension $3 g-3+k$.

A $J$-holomorphic map from a connected nodal curve is stable if the image of every unstable irreducible component of $C$ represents a non-trivial element of $H_{2}(X)$. For technical reasons it is helpful, following Ruan and Tian [RT1], [RT2] to replace (1.1) by the inhomogeneous equation

$$
\bar{\partial}_{J} f=\nu
$$

where $\nu$ is an appropriate perturbation term. With that, the space of stable maps

$$
\overline{\mathcal{M}}_{g, k}(X, A)
$$

is compact and, for generic $(J, \nu)$, is an orbifold with

$$
\operatorname{dim} \overline{\mathcal{M}}_{g, k}(X, A)=2[(n-3)(1-g)-K \cdot A]+2 k
$$

where $\operatorname{dim} X=2 n$ and $K$ is the canonical class of $(X, J)$. This space has an orientation and comes with a map

$$
\text { st } \times e v: \overline{\mathcal{M}}_{g, k}(X, A) \rightarrow \overline{\mathcal{M}}_{g, k} \times X^{k}
$$

that associates to a stable map $(f, C)$ the stabilization $s t(C)$ of its domain (obtained by collapsing all unstable irreducible components of $C$ to points) and the images $e v_{i}(f, C)=f\left(x_{i}\right)$ of the marked points. We can then pushforward the fundamental homology class of $\overline{\mathcal{M}}_{g, k}(X, A)$ by the map (1.6) and evaluating on cohomology classes $\beta \in H^{*}\left(\overline{\mathcal{M}}_{g, k}, \mathbb{Q}\right)$ and $\gamma_{1}, \ldots, \gamma_{k} \in H^{*}(X ; \mathbb{Q})$. The resulting numbers are the Gromov-Witten invariants

$$
G W_{g, k}(X, A)\left(\beta ; \gamma_{1}, \ldots, \gamma_{k}\right)=\left(\beta \cup \gamma_{1} \cup \cdots \cup \gamma_{k}\right) \cap\left((s t \times e v)_{*}\left[\overline{\mathcal{M}}_{g, k}(X, A)\right]\right) .
$$

Standard cobordism arguments show that these are independent of the choice of generic $(J, \nu)$, and hence are symplectic invariants. Once we have fixed a space $X$, we will often write $\mathrm{GW}$ invariants

$$
G W_{A, g}(X)\left(\beta ; \gamma_{1}, \ldots, \gamma_{k}\right) \quad \text { as simply } \quad G W_{A, g}\left(\beta ; \gamma_{1}, \ldots, \gamma_{k}\right) .
$$

The Gromov-Witten invariants are counts of the number of perturbed holomorphic maps that satisfy the constraints imposed by the $\beta$ and $\gamma$ classes. The $\gamma$ constraints have a simple geometric interpretation: if one chooses pseudomanifolds $V_{i} \subset X$ representing the Poincare dual of $\gamma_{i}$, then, assuming that $(J, \nu)$ is generic and that the $V_{i}$ are in general position, $G W_{A, g}\left(\gamma_{1}, \ldots, \gamma_{k}\right)$ is an oriented count of the number of $(J, \nu)$-holomorphic maps $f$ from a genus $g$ curve with $k$ marked points $x_{1}, \ldots x_{k}$ that represent the class $A$ and satisfy $f\left(x_{k}\right) \in V_{i}$ for each $i$.

This geometric interpretation implies several properties of the GW invariants. For example, the invariant vanishes unless the total degree of the constraints (the cohomology classes $\beta$ and $\gamma_{i}$ ) is equal to the dimension (1.5) of the space of stable maps. An especially useful property is the "Divisor Axiom": for any degree 2 constraint $\gamma_{1}$ with Poincaré dual $V_{1}$ we have

$$
G W_{A, g}\left(\gamma_{1}, \ldots, \gamma_{k}\right)=\left(A \cdot V_{1}\right) G W_{A, g}\left(\gamma_{2}, \ldots, \gamma_{k}\right) .
$$

This holds because, when $V_{1}$ is in general position, the image of any stable map $f: C \rightarrow X$ representing $A$ intersects $V_{1}$ transversally at $A \cdot V_{1}$ points, so there are exactly $A \cdot V_{1}$ possibilities for the first marked point.

The geometric meaning of the $\beta$ constraints is more subtle. But certain $\beta$ constraints play an important role in applications, as the examples in the next two sections show. 


\section{Splitting the Domain}

The Deligne-Mumford space $\overline{\mathcal{M}}_{g, k}$ has a complex codimension-one subset whose generic element is a stable curve with one node. Consider the case, shown below, in which the node separates $C$ into two components $C_{1}$ and $C_{2}$. The genus decomposes as $g=g_{1}+g_{2}$ and, because the node cannot be a marked point, the marked and nodal points separate into two sets $x_{1}, \ldots, x_{k_{1}+1}$ and $y_{1}, \ldots, y_{k_{2}+1}$ with $k=k_{1}+k_{2}$. There is then a map

$$
\sigma: \overline{\mathcal{M}}_{g_{1}, k_{1}+1} \times \overline{\mathcal{M}}_{g_{2}, k_{2}+1} \rightarrow \overline{\mathcal{M}}_{g, k}
$$

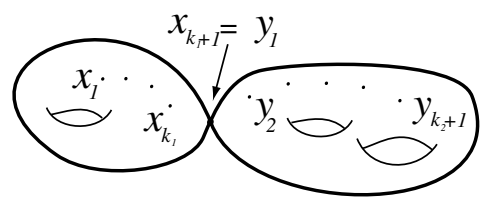

Figure 1

defined by identifying $x_{k+1}$ with $y_{1}$. We denote the Poincaré dual of the image of this map $\sigma$ by $P D(\sigma)$. Gromov-Witten invariants with constraint $P D(\sigma)$

$$
G W_{A, g}\left(P D(\sigma) ; \gamma_{1}, \cdots, \gamma_{k}\right)
$$

give counts for stable holomorphic maps from nodal domains of the type shown.

Alternatively, a map $f: C \rightarrow X$ from such a nodal domain can also be thought of as a pair of maps $f_{1}: C_{1} \rightarrow X$ and $f_{2}: C_{2} \rightarrow X$ with $f_{1}\left(x_{k_{1}+1}\right)=f_{2}\left(y_{1}\right)$. The set of such pairs $\left(f_{1}, f_{2}\right)$ can be expressed in terms of the evaluation maps

$$
e v_{k_{1}+1,1}: \overline{\mathcal{M}}_{g_{1}, k_{1}+1}\left(X, A_{1}\right) \times \overline{\mathcal{M}}_{g_{2}, k_{2}+1}\left(X, A_{2}\right) \rightarrow X \times X
$$

that record the images of the marked points $x_{k_{1}+1}$ and $y_{1}$ : the stable maps from nodal domains are those in the inverse image of the diagonal $\Delta$ under (2.2). Thus the nodal maps define a homology class

$$
\left[\overline{\mathcal{M}}_{g_{1}, k_{1}+1}\left(X, A_{1}\right)\right] \otimes\left[\overline{\mathcal{M}}_{g_{2}, k_{2}+1}\left(X, A_{2}\right)\right] \cap \Delta^{*} \quad \text { where } \quad \Delta^{*}=e v_{k_{1}+1,1}^{*}(P D[\Delta])
$$

By the gluing theorem of Ruan-Tian [RT1], this gives a second description of the Gromov-Witten invariants (2.1).

We can go one step further. Note that the class (2.3) depends only on the homology class of the diagonal in $H_{*}(X \times X ; \mathbb{Z})$. We can then use the "splitting the diagonal" formula: if $\left\{H_{a}\right\}$ and $\left\{H^{a}\right\}$ are bases of $H^{*}(X ; \mathbb{Z})$ dual by the intersection form, we have

$$
P D[\Delta]=\sum_{a} H^{a} \otimes H_{a}
$$

Inserting this in (2.3) and evaluating on classes $\gamma_{1}, \cdots, \gamma_{k}$ then yields the first splitting formula.

Proposition 2.1 (Splitting Formula) With $\sigma$ as in Figure 1 and any classes $\gamma_{1}, \cdots, \gamma_{k} \in H^{*}(X ; \mathbb{Q})$, we have

$$
\begin{aligned}
& G W_{A, g}\left(P D(\sigma) ; \gamma_{1}, \cdots, \gamma_{k}\right)= \\
& \sum_{A=A_{1}+A_{2}} \sum_{a} G W_{A_{1}, g_{1}}\left(\gamma_{1}, \cdots, \gamma_{k_{1}}, H_{a}\right) G W_{A_{2}, g_{2}}\left(H^{a}, \gamma_{k_{1}+1}, \cdots, \gamma_{k}\right) .
\end{aligned}
$$


There is a parallel story for domains with non-separating nodes. A genus $g-1$ curve with $k+2$ marked points becomes a genus $g$ curve with $k$ marked points and a single non-separating node by identifying the last two marked points. That gives a map

$$
\theta: \overline{\mathcal{M}}_{g-1, k+2} \rightarrow \overline{\mathcal{M}}_{g, k}
$$

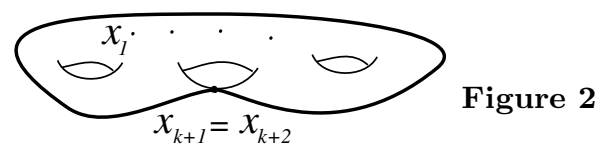

Stable maps from such genus $g$ domains are counted by GW invariants constrained by the Poincaré dual $P D(\theta)$ of the image of this map $\theta$. Alternatively, they are counted by maps $f: C \rightarrow X$ from genus $g-1$ domains with $k+2$ marked points and with $f\left(x_{k+1}\right)=f\left(x_{k+2}\right)$; this constraint is the pullback of $P D(\Delta)$ under the evaluation map

$$
e v_{k+1, k+2}: \overline{\mathcal{M}}_{g-1, k+2}(X, A) \rightarrow X \times X
$$

analogous to (2.3). Again splitting the diagonal in homology, we get the splitting formula for non-separating nodes:

$$
\begin{aligned}
G W_{A, g}\left(P D(\theta) ; \gamma_{1}, \cdots, \gamma_{k}\right) & =\sum_{a} G W_{A, g-1}\left(\gamma_{1}, \cdots, \gamma_{k}, H_{a}, H^{a}\right) \\
& =(A \cdot A) G W_{A, g-1}\left(\gamma_{1}, \cdots, \gamma_{k}\right)
\end{aligned}
$$

where the last equality holds on 4-manifolds.

The splitting formulas relate GW invariants in a given genus to invariants with smaller symplectic area or lower genus. We will see later how such relations can be encoded as differential equations on generating functions for GW invariants and used to help determine those invariants.

\section{TRR and Descendents}

GW invariants constrained by cohomology classes in $\overline{\mathcal{M}}_{g, k}$ can sometimes be rewritten and simplified using the collection of formula called the "Topological Recussion Relations" (TRR). TRR formulas will play an important role in later sections. The basic idea is the same as in the previous section: relations among cohomology classes in Deligne-Mumford spaces give rise to relations among GW invariants. Here we will explain one particular TRR formula, which involves genus 1 GW invariants constrained by "descendent classes". We also describe the geometric meaning of those constraints in simple cases. The article [Ge] of E. Getzler is a good reference for this material.

This genus 1 TRR formula originates from a fact about the space $\overline{\mathcal{M}}_{1,1}$ of complex structures on a torus with one marked point. This is an orbifold whose boundary $\overline{\mathcal{M}}_{1,1} \backslash \mathcal{M}_{1,1}$ consists of a single point, which corresponds to a curve of geometric genus $g=0$ with one non-separating node. There is a natural line bundle $\mathcal{L} \rightarrow \overline{\mathcal{M}}_{1,1}$ whose fiber at a curve $(C, x)$ is the cotangent space $T_{x}^{*} C$. The chern class of $c_{1}(\mathcal{L})$, which is usually denoted by $\phi_{1}$, is a multiple of the Poincaré dual of the orbifold fundamental class $\delta$ of the boundary: 


$$
\phi_{1} \stackrel{\text { def }}{=} c_{1}(\mathcal{L})=\frac{1}{12} \delta \quad \text { in } \quad H^{2}\left(\overline{\mathcal{M}}_{1,1} ; \mathbb{Q}\right) .
$$

There is a corresponding story on the space $\overline{\mathcal{M}}_{1,1}(X, A)$ of stable maps into $X$. There is again a line bundle $\overline{\mathcal{L}}$ whose fiber at $(C, x, f)$ is the cotangent space $T_{x}^{*} C$; its first chern class is denoted by $\psi_{1}$. By the definition of stable map, each irreducible component of the domain is either a stable curve or has non-trivial image in $X$. Under the stabilization map

$$
\text { st }: \overline{\mathcal{M}}_{1,1}(X, A) \rightarrow \overline{\mathcal{M}}_{1,1}
$$

of (1.6), the inverse image of $\delta$ consists of maps from a rational nodal curve, possibly with one or more unstable $g=0$ "bubble" components. Over the open dense set of $\overline{\mathcal{M}}_{1,1}(X, A)$ consisting of maps with irreducible domains, the relative cotangent bundles are related by $\overline{\mathcal{L}}=s t^{*} \mathcal{L}$, but that is not the case for maps with unstable components - there are correction terms. The correct relation can be written symbolically as

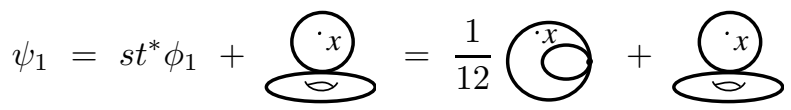

This equation requires some interpretation. The pictures represent the (real) codimension two stratum of $\overline{\mathcal{M}}_{1,1}(X, A)$ that is the closure of the set of all stable maps whose domains have the form shown; in particular the "pinched torus" in the middle picture stands for $s t^{-1}(\delta)$. With that understood, the symbolic formula (3.2) says that the GW invariant with constraint $\psi_{1}$ is equal to $\frac{1}{12}$ the invariant with constraint $\delta$ plus the contribution of the stratum whose generic element consists of stable maps from a smooth torus with one $g=0$ bubble attached.

In practice, the GW invariants constrained by $\delta$ (the middle picture) can be expressed in terms of genus 0 GW invariants using the Splitting Formula of GW invariants. The invariants constained as in the last picture can similarly be expressed in terms of invariants corresponding to the two components.

We can use $\psi_{1}$ to impose constraints on GW invariants. That is commonly done by using the evaluation map ev $: \overline{\mathcal{M}}_{1,1}(X, A) \rightarrow X$ (which simply evaluates each stable map at the given marked point) to introduce "descendent classes"

$$
\tau(\gamma)=\psi_{1} \cup e v^{*} \gamma
$$

for each cohomology class $\gamma \in H^{*}(X, \mathbb{Q})$. With more marked points, we have a similar evaluation map $e v_{i}: \overline{\mathcal{M}}_{g, k}(X, A) \rightarrow X$ for each marked point and line bundles $\overline{\mathcal{L}}_{i}$ (whose fiber at $\left(f, C, x_{1}, \ldots, x_{k}\right)$ is the cotangent space $T_{x_{i}}^{*} C$ ) and corresponding descendent classes $\tau\left(\gamma_{i}\right)=\psi_{i} \cup e v_{i}^{*} \gamma_{i}$ where $\psi_{i}=c_{1}\left(\overline{\mathcal{L}}_{i}\right)$. We then get constrained GW invariants by evaluating products of such descendent classes on the fundamental homology class of the space of stable maps. The simplest example occurs when $2+\operatorname{deg} \gamma$ is equal to the dimension of the moduli space; then the constrained invariant with one descendent

$$
G W_{g, 1}(X, A)(\tau(\gamma))=\left(\psi_{1} \cup e v^{*} \gamma\right) \cap\left[\overline{\mathcal{M}}_{g, 1}(X, A)\right]
$$

is a number.

This invariant (3.4) has a nice geometric interpretation when the Poincaré dual of $\gamma$ can be represented by a codimension two $J$-invariant submanifold $V_{\gamma} \subset X$ with trivial normal bundle (as happens in the computation of the next section). 
In that case we can fix a trivialization of the normal bundle to $V_{\gamma}$ and make two observations:

- The "cutdown moduli space" $\overline{\mathcal{M}}(\gamma) \subset \overline{\mathcal{M}}_{g, 1}(X, A)$ consisting of all maps $f$ : $(C, x) \rightarrow X$ which take the marked point $x$ onto $V_{\gamma}$ represents $e v^{*} \gamma \cap\left[\overline{\mathcal{M}}_{g, 1}(X, A)\right]$.

- For each map $f:(C, x) \rightarrow X$, the normal component $d f_{x}^{N}$ of the differential at the marked point $x$ is an element of $T_{x}^{*} C$. Thus $f \mapsto d f_{x}^{N}$ is a section of $\overline{\mathcal{L}}_{1} \rightarrow \overline{\mathcal{M}}_{g, 1}(X, A)$. The zeros of this section along $\overline{\mathcal{M}}(\gamma)$ represent the invariant (3.4) provided those zeros occur only at maps in $\overline{\mathcal{M}}(\gamma)$ that have no components with images in $V_{\gamma}$.

Thus, when this last italicized caveat holds, the descendent number (3.4) counts the number of stable maps $f$ with $f(x) \in V$ and $d f_{x}^{N}=0$, that is, those maps whose image contacts $V$ to order at least two at $f(x)$.

This story extends to higher order contact. When $f^{N}$ vanishes to order $m$ at $x=x_{1}$, the leading coefficient in its Taylor series is naturally an element of $\operatorname{Sym}^{m}\left(T_{x} C\right)$. Intrinsically, the first $m$ terms in the Taylor series is a section of the jet bundle $J^{m}(x)$, and we have the "jet exact sequence"

$$
0 \rightarrow J^{m-1}(x) \rightarrow J^{m}(x) \rightarrow \operatorname{Sym}^{m}\left(T_{x} C\right) \rightarrow 0 .
$$

Globally over the set of stable maps satisfying conditions a) and b) above, the first $m$ terms in the Taylor series give a section of a relative jet bundle $\mathcal{J}^{m}$ whose Euler class, computed from the jet exact sequence and induction, is

$$
c_{1}\left({\overline{\mathcal{L}_{1}}}^{m}\right) \cup c_{1}\left({\overline{\mathcal{L}_{1}}}^{m-1}\right) \cup \ldots \cup c_{1}\left(\overline{\mathcal{L}_{1}}\right) \cup e v_{1}^{*}(\gamma)=m ! \psi_{1}^{m} \cup e v_{1}^{*}(\gamma) .
$$

Thus it is natural to consider not just $\tau$, but also the more general descendent classes $\tau_{m}$ defined by

$$
\tau_{m}\left(\gamma_{i}\right)=\psi_{i}^{m} \cup e v_{i}^{*}\left(\gamma_{i}\right)
$$

and the corresponding descendent invariants

$$
G W_{g, k}(X, A)\left(\tau_{m_{1}}\left(\gamma_{1}\right), \ldots, \tau_{m_{k}}\left(\gamma_{k}\right)\right)
$$

which one sees in the literature. In the simplest case of a single marked point, the discussion above shows that the invariant

$$
G W_{g, 1}(X, A)\left(\tau_{m}(\gamma)\right)
$$

has a straightforward interpretation whenever conditions in a) and b) above hold: it counts the number of stable maps $f$ whose image contacts $V_{\gamma}$ to order $m+1$ at the point $f(x)$. We will use this geometric interpretation (in the case $m=1$ ) in Section 5 below.

\section{Splitting the Target: The Symplectic Sum Formula}

Another powerful compuational tool is the Symplectic Sum Formula. This can be viewed as a fact about maps into a symplectic fibration. Consider a fibration $\lambda: \mathcal{Z} \rightarrow D$ over the disk whose fibers are smooth symplectic manifolds for $\lambda \neq 0$. 


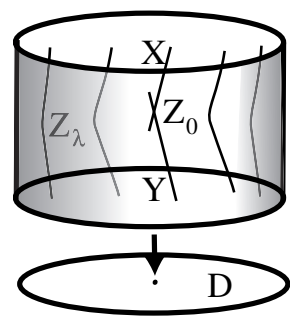

Figure 3

Suppose that the central fiber $Z_{0}$ is the singular symplectic manifold $X \cup_{V} Y$ obtained by attaching smooth symplectic manifolds $X$ and $Y$ along a common symplectic submanifold $V$ of real codimension two, as shown in Figure 3. In this situation the generic fiber $Z_{\lambda}$ is a symplectic smoothing of $Z_{0}$ and is unique up to symplectic isotopy. That isotopy class is called the symplectic sum of $(X, V)$ and $(Y, V)$.

Alternatively, one can construct symplectic sums by hand. Fix a symplectic manifold $X$ with a symplectic codimension two submanifold $V$. Suppose we are given a similar pair $(Y, V)$ with a symplectic identification between the two copies of $V$ such that, under that identification, the normal bundles $N_{X}^{V}$ and $N_{Y}^{V}$ are dual as complex vector bundles. We can then glue $X$ to $Y$ along $V$ and "round the corner", essentially by replacing the crossing $x y=0$ by $x y=\lambda$ in coordinates normal to $V$. For $\lambda \neq 0$ the resulting manifold $Z_{\lambda}$ is smooth and symplectic this is the symplectic sum. In fact, this construction creates a family $\left\{Z_{\lambda}\right\}$ that together form a symplectic fibration as above.

The "Symplectic Sum Formula" developed in [IP2] and [IP3] expresses the GW invariants of the symplectic sum $Z_{\lambda}$ in terms of invariants of $X$ and $Y$. The derivation begins by considering what happens to $J$-holomorphic maps into $Z_{\lambda}$ as $\lambda \rightarrow 0$. By the compactness theorem for $J$-holomorphic maps (for maps into $\mathcal{Z}$ ), these limit to maps into $Z_{0}=X \cup_{V} Y$, which can be separated into components on the $X$ and $Y$ sides. However, there are several complications.

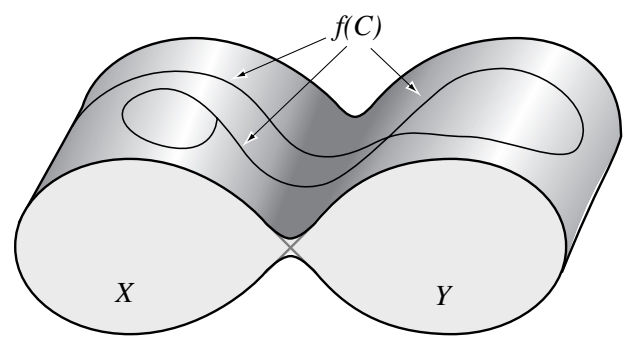

Figure 4 Images $f(C)$ of $J$-holomorphic maps into $Z_{\lambda}$ as $\lambda \rightarrow 0$.

One complication is apparent in Figure 4: connected curves in $Z_{\lambda}$ may limit to curves whose restrictions to $X$ and $Y$ are not connected, as Figure 4 shows. For that reason the GW invariant, which counts stable curves from a connected domain, is not the appropriate invariant for expressing a sum formula. Instead one should work with the "Gromov-Taubes" invariant $G T$, which counts stable maps from domains that need not be connected. This is not a substantive change: one can move between the GW invariants and the GT invariants contain the same information and there are combinatorial formulas relating them.

A more substantial complication arises from the requirement, needed for the compactness theorem mentioned above, that the the almost complex structures on $X$ and $Y$ match along $V$ and extend smoothly to the fibers of the fibration $\mathcal{Z}$. 
For that reason, it is necessary to restrict to pairs $(J, \nu)$ that satisfy a certain " $V$ compatibility" condition (see [IP3]). That condition implies, in particular, that $V$ is a $J$-holomorphic submanifold. There is a price to pay for this specialization. Because we can no longer perturb $(J, \nu)$ along $V$ at will, we do not have enough freedom to arrange that the limit curves are transverse to $V$. In fact, the images of the limit maps into $Z_{0}$ can meet $V$ at points with multiplicities and, worse, may have components may be mapped entirely into $V$.

To count stable maps into $Z_{0}$ we look first at maps into $X$ that have no components mapped into $V$. These form a moduli space which is the union of components $\mathcal{M}_{s}^{V}(X)$ labeled by the multiplicities $s=\left(s_{1}, \ldots, s_{\ell}\right)$ of the intersection points with $V$. Each $\mathcal{M}_{s}^{V}(X)$ can be compactified. Those compact moduli spaces come with evaluation maps like (1.6) that keep track of the domain curve, the images of the marked points, and the relative homology class of the image (details are given in [IP2]). Repeating the construction of GW invariants, we then obtain Relative Gromov-Witten invariants $G W_{X}^{V}$ that count the number of stable maps into $X$ that meet $V$ with specified multiplicities.

A third complication is the fact that the squeezing process is not injective. A precise analysis reveals that each map into $Z_{0}$ that meets $V$ with multiplicities $s=\left(s_{1}, \ldots, s_{\ell}\right)$ is the limit of $s_{1} \cdot s_{2} \cdots s_{\ell}$ distinct maps into $Z_{\lambda}$. The maps within such a "cluster" have almost the same image but have different domains. As $\lambda \rightarrow 0$ the cluster coalesces, limiting to a single map.

The limit process is reversed by a standard approach that goes back to Taubes and Donaldson: construct a space of approximately holomorphic maps (which incorporates the clustering phenomenon), then correct the approximate maps to true holomorphic maps using a fixed point theorem. The upshot is a precise statement, at the level of moduli spaces, of the fact that stable maps into $Z_{\lambda}$ for small $\lambda$ are in one-to-one correspondence to the clusters associated with pairs of maps $(f, g)$ into $X$ and $Y$ that intersect $V$ at the same points with the same multiplicities.

There remains the issue of curves "sinking into the neck" in the limit $\lambda \rightarrow 0$. This is dealt with using a renormalization argument. For that, we consider the ruled manifold $\mathbb{P}_{V}=\mathbb{P}\left(\mathbb{C} \oplus N_{X}^{V}\right)$, which is the $\mathbb{P}^{1}$ bundle over $V$ obtained by adding an infinity section to the normal bundle to $V$. We can then glue $X$ to $Y$ through a series of $n$ copies of $\mathbb{P}_{V}$, obtaining a space which is singular along $n+1$ copies of $V$. Introducing smoothing parameters $\lambda_{1}, \ldots, \lambda_{n+1}$ and applying the symplectic sum construction gives a fibration $\mathcal{Z}_{n} \rightarrow D^{n+1}$. This is similar to the fibration of Figure 3 , but now the fibers near the central fiber are manifolds $Z_{\lambda}$ with $n+1$ necks, as shown in Figure 5 for $n=2$. An energy bound shows that, when one takes $n$

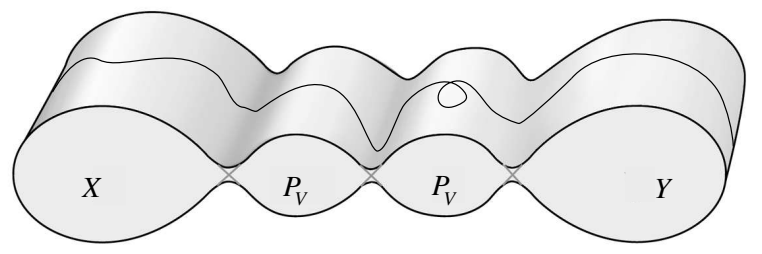

Figure 5 Renormalizing along $V$ several times catches prevents curves from sinking into the neck. 
sufficiently large and lets $\lambda \rightarrow 0$, the limit maps have no components sinking into most necks of $Z_{0}$. Squeezing some or all of those necks decomposes the curves in $Z_{\lambda}$ into curves in $X$ joined to curves in $Y$ by a chain of curves in intermediate spaces $\mathbb{P}_{V}$, and the previous analysis applies to each. Working through the combinatorics, one finds that the total contribution of the entire region between $X$ and $Y$ is given by a certain $G T$ invariant $S_{V}$ of $\mathbb{P}_{V}$. This " $S$-matrix" $S_{V}$ keeps track of how the genus, homology class, and intersection points with $V$ are "scattered" as the images of stable maps pass through the intermediate regions of Figure 5.

Symplectic Sum Theorem Let $Z$ be the symplectic sum of $(X, V)$ and $(Y, V)$. Then the Gromov-Taubes invariant of $Z$ is given in terms of the relative invariants of $X$ and $Y$ by

$$
G T_{Z}=G T_{X}^{V} * S_{V} * G T_{Y}^{V}
$$

where $*$ is a certain 'convolution operation' and $S_{V}$ is a certain $G W$ invariant of the ruled manifold $\mathbb{P}_{V}$.

\section{Application: Invariants of $E(1)$}

The formulas of the previous sections describe the behavior of GW invariants under various cut-and-paste operations. We referred to those as "compuational tools", but have not yet done any computations or shown that they have practical value. We now remedy that by presenting a striking application: calculating GW invariants for the rational elliptic surface $E(1)$.

$E(1)$ is the simplest Kähler surface that fibers over the 2-sphere with elliptic curves as fibers (that is, the fibers are tori with varying complex structure). One standard construction goes as follows. Choose two generic degree 3 curves in $\mathbb{P}^{2}$. These are elliptic curves, given as the zero sets of cubic polynomials $f, g$ in homogeneous coordinates, which intersect at 9 points. Blowing up $\mathbb{P}^{2}$ at those 9 points gives the surface $E(1)$. It comes with 9 exceptional curves. Furthermore, the curves in the linear system $\{f+t g \mid t \in \mathbb{C}\}$ lift to a family of elliptic curves $\left\{E_{t}\right\}$ in $E(1)$ so that (a) $E_{t}$ and $E_{t^{\prime}}$ are disjoint for $t \neq t^{\prime}$, (b) every point of $E(1)$ lies on one and only $E_{t}$, and (c) each $E_{t}$ intersects each exceptional curve in one point with multiplicity one. Thus the $E_{t}$ are the fiber of a fibration $E(1) \rightarrow S^{2}$ and each exceptional curve is a section of that fibration. For generic cubic polynomials $f$ and $g$, this fibration has exactly 12 nodal fibers as shown in Figure 6 below.

Varying the blowup points gives different complex structures on $E(1)$ but, up to isotopy, there is a single symplectic structure on $E(1)$. The homology $H_{2}(E(1) ; \mathbb{Z})$ is generated by the 9 section classes $S_{i}$ represented by the exceptional curves and by the class $F$ of a generic fiber. These satisfy

$$
F \cdot F=0, \quad F \cdot S_{i}=1, \quad S_{i} \cdot S_{j}=-\delta_{i j},
$$

and altogether the intersection form of $E(1)$ is the sum $E_{8} \oplus H$ of the $E_{8}$ matrix and the $2 \times 2$ hyperbolic matrix. The adjunction formula shows that the canonical class is $K=-F$. The GW invariant of the fiber class is especially important: it is

$$
G W_{F, 1}(E(1))=1
$$

(see Example 9.3 of [IP2]). 
Now fix one section $S=S_{i}$ and consider the classes $S+d F$ for $d \in \mathbb{Z}$. For generic $(J, \nu)$, the space of stable maps $S^{2} \rightarrow E(1)$ form a moduli space with dimension given by (1.5):

$$
\operatorname{dim} \overline{\mathcal{M}}_{0,0}(E(1), S+d F)=2[-1-K \cdot(S+d F)]=0 .
$$

Thus for each $d$ this moduli space is a finite set of points (with sign). We wish to compute the GW invariants that count the signed points in those moduli spaces.

Problem A Find the genus 0 Gromov-Witten invariants $G W_{S+d F, 0}$ of $E(1)$. Equivalently, find the generating function

$$
F(t)=\sum_{d \geq 0} G W_{S+d F, 0} t^{d} .
$$

Notice that any class $S+d F$ with $G W_{S+d F, 0} \neq 0$ is represented by a $(J, \nu)$ holomorphic map for generic $(J, \nu)$. As $\nu \rightarrow 0$ those maps limit to a $J$-holomorphic map representing $S+d F$. By also varying $J$, we see that $S+d F$ has a holomorphic representative for the $J$ of a complex elliptic structure on $E(1)$, for which we know that $S$ also has a holomorphic representative. Since distinct holomorphic curves have non-negative intersection we conclude that $d=0$ or $d-1=S \cdot(S+d f) \geq 0$. Thus there is no lose of generality in restricting the above sum to $d \geq 0$.

There is a similar problem for genus 1 invariants. Note that, by (1.5), the space of stable maps from a genus $g=1$ domain into $E(1)$ has dimension 2 . We can get a GW invariant by adding a marked point (thereby increasing the dimension of space of stable maps to 4 ) and imposing the codimension 4 constraint $\tau(F)$. For that purpose, fix a smooth generic fiber $F_{0} \subset E(1)$.

Problem B Find the genus $1 \mathrm{GW}$ invariants $G W_{S+d F, 1}(\tau(F))$ of $E(1)$. Equivalently, find the generating function

$$
H(t)=\sum_{d \geq 0} G W_{S+d F, 1}(\tau(F)) t^{d} .
$$

We will solve Problems A and B simultaneously by relating the generating functions in two ways: using a TRR formula and using the Symplectic Sum formula. That will give two differential equations involving $F(t)$ and $H(t)$ that, together, determine $F(t)$ and $H(t)$ explicitly.

First, we can expand the constraint $\tau(F)=\psi_{1} \cup e v^{*}(F)$ using the TRR formula (3.2):

$$
G W_{S+d F, 1}(\tau(F))=\frac{1}{12} \widehat{\Omega}^{F_{0}}+\widehat{x}^{F_{0}}
$$

where the arrows to $F_{0}$ indicate that the marked points must be mapped onto $F_{0}$. The pinched torus picture corresponds to a genus 0 domain with automorphism group $\mathbb{Z}_{2}$. When that is expanded using (2.5), summed over $d$ and simplified using the Divisor Axiom, we have

$$
\begin{aligned}
\frac{1}{12} \cdot \frac{1}{2} \sum_{d \geq 0}(S+d F)^{2} G W_{S+d F, 0}(F) t^{d} & =\frac{1}{24} \sum_{d \geq 0}(2 d-1) G W_{S+d F, 0}(F) t^{d} \\
& =\frac{1}{24}\left(2 t F^{\prime}(t)-F(t)\right)
\end{aligned}
$$


Maps corresponding to the last picture of (5.1) have components $f_{1}: S^{2} \rightarrow E(1)$ and $f_{2}: T^{2} \rightarrow E(1)$ that together represent $S+d F$. Composing with $\pi: E(1) \rightarrow S^{2}$ gives holomorphic maps $\pi \circ f_{i}$ to $S^{2}$ whose total degree is 1 . This implies that one of the maps $\pi \circ f_{i}$ has degree 0 , so its image is a single point. Thus either $f_{1}$ or $f_{2}$ is a map into a single fiber of $E(1)$. But that cannot be the case for $f_{1}$ because $f_{1}$, being a stable map with unstable domain, must represent a non-trivial homology class and there are no homologically non-trivial maps $S^{2} \rightarrow T^{2}$. We conclude that $f_{2}$, if not trivial, is a cover of a $J$-holomorphic fiber — which must be $F_{0}$ - and represents $d_{2} F$, while $f_{1}$ represents $S+d_{1} F$ with $d_{1}+d_{2}=d$.

Now applying Proposition 2.1 and the Divisor Axiom, we see that the maps corresponding to the last picture of (5.1) contribute

$$
\begin{aligned}
& \sum_{d \geq 0} t^{d} \sum_{d_{1}+d_{2}=d} \sum_{a} G W_{S+d_{1} F, 0}\left(F, H_{a}\right) \cdot G W_{d_{2} F, 1}\left(H^{a}\right) \\
= & \sum_{d \geq 0} t^{d}\left(\sum_{k>0} G W_{S+(d-k) F, 0} \cdot k G W_{k F, 1}+\sum_{a} G W_{S+d F}\left(H_{a}\right) G W_{0,1}\left(H^{a}\right)\right) \\
= & F(t) \sum_{d>0} d G W_{d F, 1} t^{d}+\sum_{d \geq 0} t^{d} \sum_{a} G W_{S+d F}\left(H_{a}\right) G W_{0,1}\left(H^{a}\right) .
\end{aligned}
$$

The invariant $G W_{0,1}\left(H^{a}\right)$ is a count of degenerate maps and one can show by hand that

$$
G W_{0,1}\left(H^{a}\right)=\frac{1}{24}\left(H^{a} \cdot K\right)=-\frac{1}{24}\left(H^{a} \cdot F\right)
$$

( see [IP3] $\S 15$ ). The invariant $d G W_{d F, 1}$ counts the $d$-fold coverings of a torus by tori and hence it is the sum of all divisors of $d$, which is denoted by $\sigma(d)$. We set

$$
G(t)=\sum_{d=1}^{\infty} \sigma(d) t^{d}=\sum_{k=1}^{\infty} \frac{k t^{k}}{1-t^{k}}
$$

Then, combining the last several equations yields a differential equation that encodes the "splitting the domain" TRR relation:

$$
H(t)=\frac{1}{12}\left(t F^{\prime}(t)-F(t)\right)+G(t) F(t)
$$

We will next derive a similar differential equation by splitting the target. Let $V$ be one of the fibers of $E(1) \rightarrow S^{2}$. Setting $E(0)=S^{2} \times T^{2}$, let $V^{\prime}$ be a fiber of $E(0) \rightarrow S^{2}$. Since $V$ and $V^{\prime}$ have trivial normal bundles, we can identify them and form the symplectic sum. That allows us to regard $E(1)$ as $E(1) \# E(0)$, as in Figure 6. We will apply the symplectic sum formula (4.1), taking the constraint $\tau(F)$ with $F$ represented by a fiber $F_{0}$ on the $E(0)$ side. 


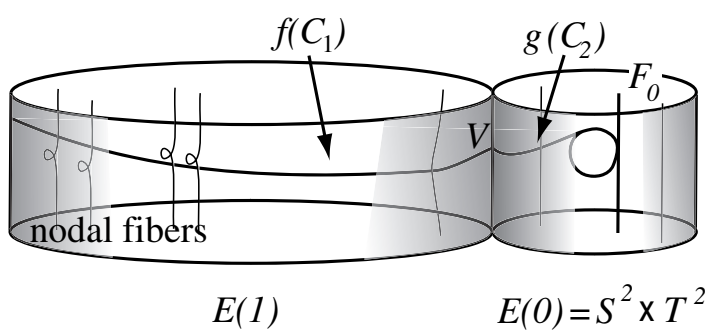

Figure 6

Pinching $E(1) \#_{V} E(0)$

In this case the symplectic sum formula simplifies considerably. To see how, one should think about the proof, rather than the formula itself. As we squeeze the neck in Figure 6 by letting $\lambda \rightarrow 0$, maps from genus 1 domains that represent $S+d F$ limit to maps $f: C_{1} \rightarrow E(1)$ and $g: C_{2} \rightarrow E(0)$ with (genus $\left.C_{1}\right)+$ (genus $\left.C_{2}\right)=1$ and whose images meet at a point of $V$ and with the image of $g$ contacting $F_{0}$ in a way that satisfies the constraint $\tau(F)$.

First consider the possibility that $C_{2}$ has genus 0 . Then $g\left(C_{2}\right)$ represents $S+d^{\prime} F$ for some $d^{\prime}$, where $S$ and $F$ are the section and fiber classes of $E(0)$. Composing $g$ with the projection $E(0) \rightarrow T^{2}$ gives a map $S^{2} \rightarrow T^{2}$, which must be zero in homology, so $d^{\prime}=0$. Stability implies that $C_{2}$ is a smooth 2 -sphere representing $S$. Furthermore, $F_{0}$ has trivial normal bundle and the image of $g$ has no components in $F_{0}$. As we saw in Section 3, in that situation $\tau(F)$ is the constraint that the image of $g$ contacts $F_{0}$ to second order. But $S \cdot F=1$, so that is impossible for a fibered complex structure on $E(0)$. A limiting argument shows that the same is true for generic $J$, so $G W_{S, 0}(\tau(F))=0$. We conclude that $C_{1}$ has genus 0 and $C_{2}$ has genus 1.

Exactly the same argument shows that the limit curve cannot have any curves in the middle regions shown in Figure 5. Consequently, the matrix $S_{V}$ in the symplectic sum formula (4.1) is the identity and the relative and absolute invariants are the same. Furthermore, since the maps intersect $V$ at a single point with multiplicity 1 the domains $C_{1}$ and $C_{2}$ are connected and the multiplicity issues mentioned in Section 4 do not arise, and the convolution in (4.1) is simply the product of the generating functions. Since $C_{1}$ has genus 0 the generating function on the $E(1)$ side is just $F(t)$. Therefore, we have

$$
H(t)=F(t) \sum_{d \geq 0} G W_{S+d F, 1}(E(0))(\tau(F)) t^{d}
$$

We can calculate the generating function for $E(0)$ by applying TRR exactly as in (5.2) and (5.3). In fact, since $S^{2}=0$ the contribution of the first picture of (5.1), given by the left-hand side of (5.2), is zero. Moreover, the invariant $G W_{S+d F, 0}(E(0))=0$ for $d>0$, so the contribution of the second picture of (5.1), is given by the last line of (5.3) with $F(t)$ replaced by $G W_{S, 0}(E(0))=1$. That is evaluated using (5.4), noting that the canonical class of $E(0)$ is $-2 F$, and again recognizing that $d G W_{d F, 1}(E(0))$ is twice the number of $d$-fold covers of a torus by a torus (since, for generic $J, E(0)$ has exactly two holomorphic fibers in the fiber class). Consequently,

$$
\sum_{d \geq 0} G W_{S+d F, 1}(E(0))(\tau(F)) t^{d}=-\frac{1}{12}+2 G(t) .
$$


With that, the symplectic sum formula corresponding to the decomposition of Figure 6 has the simple form

$$
H(t)=-\frac{1}{12} F(t)+2 G(t) F(t) .
$$

Now the punch line: equating formulas (5.6) and (5.8) gives the ODE

$$
t F^{\prime}(t)=12 G(t) F(t)
$$

with the initial condition $F(t)=G W_{S, 0}=1$. The solution of this ODE is given by

$$
F(t)=\prod_{d \geq 1}\left(\frac{1}{1-t^{d}}\right)^{12}
$$

and we can then obtain $H(t)$ from (5.8). With one more application of the sum formula we can obtain the corresponding generating function for curves of each genus $g>0$ in $E(1)$ (see [IP1]).

\section{Enumerative Conjectures and the Inadequacy of GW Invariants}

The calculation done in Section 5 is very encouraging: we successfully used symplectic gluing methods determine GW invariants in an interesting case, and the result automatically appeared in the elegant form (5.9). Moreover, the result correctly enumerates curves in a case - rational curves in the classes $S+d F$ in $E(1)$ - of interest to algebraic geometers. Obviously, one would like to extend this approach and develop it into a general method for calculating GW invariants for whole classes of symplectic manifolds. One can further hope to relate those GW invariants to enumerative invariants of Kähler surfaces.

This idea is especially appealing because it seems to be linked to some remarkable conjectures in enumerative algebraic geometry (see [Gö]). These arose from the observation that in the cases where enumerative invariants have been proved or conjectured (some by algebraic geometers and some by string theorists), the generating functions are remarkably similar. They are always products of a few special functions. Two of those special functions, namely $F(t)$ in $(5.9)$ and $G(t)$ in (5.5), arose naturally in gluing computations of the previous section. Thus it is reasonable to conjecture that there are similar universal formulas for the GW invariants of Kähler surfaces.

But there is a catch. This vision of simple, calculable and non-trivial GW generating functions is not true for the usual Gromov-Witten invariants. In fact, for Kähler surfaces with geometric genus $p_{g}>0$ all of the $G W$ invariants of interest vanish. This phenomenon has a simple explanation, which we describe in this section. The discussion here is preparation for introducing the "family GW invariants" in the next section.

Recall that the geometric genus $p_{g}$ of a Kähler surface is the (complex) dimension of the space $H^{0,2}(X)$ of holomorphic $(0,2)$-forms. The role of $p_{g}$ can already be seen in the following example, which involves the elliptic surface $E(n)$ obtained as the $n$-fold fiber sum of copies of $E(1)$ as shown in Figure 7 .

Example 6.1 Let $E(n) \rightarrow S^{2}$ be an elliptic surface with a section of selfintersection number $-n$. This is a simply-connected Kähler manifold with a fiber 


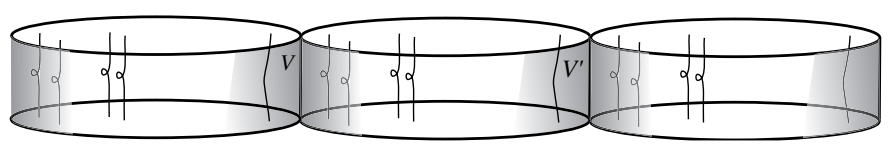

Figure $7 \quad E(3)=E(1) \#_{V} E(1) \#_{V^{\prime}} E(1)$

class $F$ and a section class $S$ satisfying $S \cdot S=-n$. Using the adjunction formula and Riemann-Roch, one sees that the canonical class is $K=(n-2) F$ and the geometric genus is $p_{g}=(n-1)$. Then by $(1.5)$ the dimension of the GW moduli space for the class $S+d F$ with genus $g=0$ and no marked points is

$$
\operatorname{dim} \overline{\mathcal{M}}_{g, 0}(E(n), S+d F)=2(-K \cdot(S+d F)-1)=2(1-n)=-2 p_{g} .
$$

This is negative when $n \geq 2$, meaning that the moduli space is empty for generic $J$. Thus the genus $0 \mathrm{GW}$-invariants for the class $S+d F$ are all zero.

More generally, in a Kähler surface $X$, the space of holomorphic curves representing a fixed homology class $A$ forms a space called the (generalied) Severi variety (see [BL3]). When the Severi variety has dimension $n$, one can impose $n$ point constraints by introducing marked points $x_{1}, \ldots x_{n}$ on the domain and $n$ points $y_{1}, \ldots y_{n}$ in $X$ and restricting attention to the set of maps $f$ with $f\left(x_{j}\right)=y_{j}$ for $j=1, \ldots n$. When certain tranversality conditions are met, that will be a finite set of maps, and count of those maps is an enumerative invariant of $X$.

However, when $p_{g}>0$ that enumerative invariant is not a GW invariant. The reason is that the (formal) dimension of the GW moduli space is strictly less than the dimension of the Severi variety. Here is a specific computation.

Example 6.2 Let $X$ be a Kähler surface with canonical class $K$. Suppose that $f: C \rightarrow X$ is an embedded holomorphic map representing a class $A$ with $A-K$ ample. Then the space $\mathcal{V}_{A}$ of curves representing $A$ has complex dimension

$$
\operatorname{dim}_{\mathbb{C}} \mathcal{V}_{A}=d_{A}+p_{g}
$$

where $d_{A}$ is the dimension (1.5) of the GW moduli space of maps representing $A$.

Proof Let $\xi$ the line bundle over $X$ corresponding to the divisor $f(C)$ and let $N$ be the normal bundle. Fixing a section with divisor $f(C)$ gives a sheaf exact sequence $0 \rightarrow \mathcal{O}_{X} \rightarrow \xi \rightarrow N \rightarrow 0$, which induces the long exact sequence

$$
\cdots \rightarrow H^{1}(X ; \xi) \rightarrow H^{1}(C ; N) \rightarrow H^{2}\left(X ; \mathcal{O}_{X}\right) \rightarrow H^{2}(X ; \xi) \rightarrow 0 .
$$

But since $A-K$ is ample, it follows from Serre duality and Kodaira Vanishing Theorem that $H^{1}(X ; \xi)$ and $H^{2}(X ; \xi)$ both vanish. Thus $H^{1}(C ; N) \cong H^{0,2}(X)$ has complex dimension $p_{g}$. On the other hand, the tangent space to $\mathcal{V}_{A}$ is the space $H^{0}(C, N)$ of holomorphic deformations of $f(C)$. Finally, by Riemann-Roch and $(1.5)$

$$
d_{A}=H^{0}(C, N)-H^{1}(C, N)=\operatorname{dim}_{\mathbb{C}} \mathcal{V}_{A}-p_{g}
$$

The situation in Example 6.2 is built into the algebraic geometry viewpoint of Gromov-Witten invariants. Ignoring technical difficulties, the picture is as follows. On a Kähler surface, the space of stable maps $\overline{\mathcal{M}}_{g, k}(X, A)$ (which is the Severi 
variety when the maps are embeddings) is a compact projective variety of some dimension $n$. There is a rank $p_{g}$ bundle $\mathcal{E}$ over that space

$$
\begin{gathered}
\mathcal{E} \\
\downarrow \\
\overline{\mathcal{M}}_{g, k}(X, A)
\end{gathered}
$$

whose fiber over a map $f$ is $H^{0,1}\left(C ; f^{*} N\right)$. The Euler class of $\mathcal{E}$ defines a homology class

$$
\overline{\mathcal{M}}_{g, k}^{\text {virt }}(X, A) \stackrel{\text { def }}{=}\left[\overline{\mathcal{M}}_{g, k}(X, A)\right] \cap e(\mathcal{E}) \in H_{2\left(n-p_{g}\right)}\left(\mathcal{V}_{A}\right)
$$

called the virtual moduli cycle. Algebraic geometers define $\mathrm{GW}$ invariants using this in place of the space (1.4) of perturbed holomorphic maps.

To relate this picture to the geometric analysis viewpoint, consider a perturbation $\nu$ as in (1.3). At each map $f$, the linearization of the $J$-holomorphic map equation is an operator $L_{f}: \Omega^{0}\left(f^{*} T X\right) \oplus H^{0,1}(T C) \rightarrow \Omega^{0,1}\left(f^{*} T X\right)$ (see [RT2]). The projection of $\nu$ into the cokernel $L_{f}$ defines a section of $\mathcal{E} \rightarrow \overline{\mathcal{M}}_{g, k}(X, A)$. The zeros of that section are exactly the $J$-holomorphic maps $f$ that, by the Implicit Function Theorem, can be uniquely corrected to solutions of the perturbed holomorphic map equation $\bar{\partial}_{J} f=t \nu$ for small fixed $t$. That zero set thus represents the virtual moduli cycle (6.2) and is homologous to the moduli space (1.4) defined by the perturbed equations.

The distinction between the GW and the enumerative counts should now be clear. Enumerative geometers impose constraints and count curves. That is in principle the same as a count of constrained maps in the space $\overline{\mathcal{M}}_{g, k}(X, A)$ of stable maps for a Kähler $J$. But it is entirely different from the GW invariants, which count constrained maps in the codimension $p_{g}$ subspace $\overline{\mathcal{M}}_{g, k}^{\text {virt }}(X, A)$, or equivalently in the space of perturbed holomorphic maps for generic $(J, \nu)$.

This brings us to the key point: to get at the enumerative invariants we need a new version of GW invariants. Those invariants should be based on a moduli space of $J$-holomorphic maps that

- is equal to the space of stable maps for a Kähler $J$, and

- has dimension $2 p_{g}$ greater than the dimension of the usual GW moduli space.

There are several approaches to doing that. Bryan and Leung [BL1],[BL2] defined and calculated such invariants for K3 and Abelian surfaces by using the twistor family. Behrend-Fantechi $[\mathrm{BF}]$ have defined invariants for a more general class of algebraic surfaces using algebraic geometry. Here we will focus on the analytic approach of the first author. That is described in the next section.

\section{$7 J_{\alpha}$-holomorphic Maps on Kähler surfaces}

This section describes the first author's family GW invariants for Kähler surfaces. These are based on the observation that, associated to each Kähler structure $J$, there is a $2 p_{g}$-dimensional family of almost complex structures, very naturally parameterized by $H^{0,2}(X)$. While this parameter space is non-compact, one can show that in many interesting cases the resulting family moduli space is compact, 
and hence defines invariants. Details of the construction outlined here can be found in [L1].

Fix a compact Kähler surface $(X, \omega, J, g)$ and choose the $2 p_{g}$-dimensional parameter space

$$
\mathcal{H}=\operatorname{Re}\left(H^{2,0} \oplus H^{0,2}\right) .
$$

Using the metric, each $\alpha \in \mathcal{H}$ defines an endormorphism $K_{\alpha}$ of $T X$ by the equation

$$
\left\langle u, K_{\alpha} v\right\rangle=\alpha(u, v) .
$$

It follows that $K_{\alpha}$ is skew-symmetric and anti-commutes with $J$. For each map $f$ : $C \rightarrow X$, we then have a $2 p_{g}$ dimensional family of elements $K_{\alpha} \partial f j$ in $\Omega^{0,1}\left(f^{*} T X\right)$. We modify the $J$-holomorphic map equation (1) by considering the pairs $(f, \alpha)$ satisfying

$$
\bar{\partial}_{J} f=K_{\alpha} \partial f j .
$$

We can recast this as a family of pseudo-holomorphic maps parameterized by $\mathcal{H}$. The above definitions imply that $I d+J K_{\alpha}$ is injective, and hence invertible, for each $\alpha \in \mathcal{H}$. Thus

$$
J_{\alpha}=\left(I d+J K_{\alpha}\right)^{-1} J\left(I d+J K_{\alpha}\right)
$$

is a family of almost complex structures on $X$ parameterized by $\alpha$ in the $2 p_{g^{-}}$ dimensional linear space $\mathcal{H}$. Some straightforward algebra shows that (7.1) is equivalent to

$$
\bar{\partial}_{J_{\alpha}} f=0 .
$$

Thus for each $J$ we get a family of holomorphic maps consisting of the pairs $(f, \alpha)$ where $f: C \rightarrow X$ is a map from a stable curve to $X$ and $\alpha \in \mathcal{H}$ which satisfy (7.1) or equivalently (7.2). Fixing the genus $g$ and number $k$ of marked points on the domain and a class $A \in H_{2}(X ; \mathbb{Z})$, the set of such pairs forms a moduli space

$$
\overline{\mathcal{M}}_{g, n}^{\mathcal{H}}(X, A, J)=\left\{(f, \alpha) \mid \bar{\partial}_{J_{\alpha}} f=0, \alpha \in \mathcal{H},[f]=A\right\}
$$

whose formal dimension

$$
\operatorname{dim} \overline{\mathcal{M}}_{g, k}^{\mathcal{H}}(X, A)=2\left[(g-1)-K \cdot A+k+p_{g}\right]
$$

is $2 p_{g}$ larger than the dimension (1.5) of the usual GW moduli space. As in ([RT1]), we can add a perturbation term to the equation (7.1) to make the moduli space an orbifold of this dimension. Then, following the standard procedure described in Section 1, we can pushforward by the evaluation and obtain symplectic invariants provided the modui space is compact.

Compactness is an issue because the parameter $\alpha$, which ranges over the vector space $\mathcal{H}$, may be unbounded. Nevertheless, one can use specific geometric arguments to show that the moduli space is compact for many classes $A$, and consequently the invariants are well-defined for those classes. In [L1], we proved that is true for many cases important in algebraic geometry.

Theorem 7.1 ([L1]) The family $G W$ invariants $G W_{A, g}^{\mathcal{H}}$ are well-defined

(a) for all non-zero classes $A$ on a K3 or Abelian surface.

(b) for all $(1,1)$ classes $A$ on a minimal elliptic surface of Kodaira dimension 1 satisfying $A \cdot($ fiber class $)>0$, and

(c) for many A on a Kähler surface of general type (specifically, for (1,1) classes $A$ that are not linear combinations of components of the canonical class $K$ ). 
For K3 and abelian surfaces, our family GW-invariants are identical to the "twistor family invariants" used by Bryan and Leung. In particular, (i) the invariants are independent of complex structures, (ii) they count actual holomorphic curves representing primitive classes, and (iii) for any two primitive classes $A$ and $B$ with $A^{2}=B^{2}$ there is a orientation preserving diffeomorphism $h: X \rightarrow X$ such that $h_{*} A=B$ and

$$
G W_{m A, g}^{\mathcal{H}}\left(h^{*}\left(\gamma_{1}\right), \cdots, h^{*}\left(\gamma_{k}\right)\right)=G W_{m B, g}^{\mathcal{H}}\left(\gamma_{1}, \cdots, \gamma_{k}\right) .
$$

\section{Splitting Formulas for Family Invariants}

One can extend the splitting formulas of Section 2 to the family GW invariants by exploiting the fact that the parameter space $\mathcal{H}$ is a linear space. For simplicity, we will describe the extension of the splitting formula only for the case of elliptic surface $E(n)$ with the classes $S+d F, d \in \mathbb{Z}$. The general case is done in [L1].

Let $\sigma$ be the gluing map of Figure 1. Each $J_{\alpha}$-holomorphic map whose domain lies in the image of $\sigma$ can be written as a pair of $J_{\alpha}$-holomorphic maps $\left(f_{1}, \alpha\right)$ : $C_{1} \rightarrow X$ and $\left(f_{2}, \alpha\right): C_{2} \rightarrow X$ with $f_{1}\left(x_{k_{1}+1}\right)=f_{2}\left(y_{1}\right)$. Now it is a basic fact, proved in [L1], that any solution of $(7.1)$ representing a $(1,1)$ class has $\alpha=0$. Since $S+d F$ is a $(1,1)$ class with respect to the complex structure of $E(n)$, both $f_{1}$ and $f_{2}$ are holomorphic. Thus, either $\left[f_{1}\right]=S+d_{1} F$ and $\left[f_{2}\right]=d_{2} F$ or $\left[f_{1}\right]=d_{1} F$ and $\left[f_{2}\right]=S+d_{2} F$ with $d_{1}+d_{2}=d$. Define

$$
\begin{aligned}
& p_{1, d_{1}, d_{2}}: \overline{\mathcal{M}}_{g_{1}, k_{1}+1}^{\mathcal{H}}\left(E(n), S+d_{1} F\right) \times \overline{\mathcal{M}}_{g_{2}, k_{2}+1}^{\mathcal{H}}\left(E(n), d_{2} F\right) \rightarrow \mathcal{H} \times \mathcal{H} \\
& p_{2, d_{1}, d_{2}}: \overline{\mathcal{M}}_{g_{1}, k_{1}+1}^{\mathcal{H}}\left(E(n), d_{1} F\right) \times \overline{\mathcal{M}}_{g_{2}, k_{2}+1}^{\mathcal{H}}\left(E(n), S+d_{2} F\right) \rightarrow \mathcal{H} \times \mathcal{H}
\end{aligned}
$$

by $p_{i, d_{1}, d_{2}}\left(\left(f_{1}, \alpha_{1}\right),\left(f_{2}, \alpha_{2}\right)\right)=\left(\alpha_{1}, \alpha_{2}\right)$ for $i=1,2$. Then the moduli space of $J_{\alpha^{-}}$ holomorphic maps $(f, \alpha)$ from nodal domains lying in $\operatorname{Im}(\sigma)$ defines a homology class

$$
\sum_{i=1,2} \sum_{d=d_{1}+d_{2}}\left(\left[p_{i, d_{1}, d_{2}}^{-1}\left(\Delta^{\mathcal{H}}\right)\right] \cap \Delta^{*}\right)
$$

where $\Delta^{\mathcal{H}}$ is the diagonal in $\mathcal{H} \times \mathcal{H}$ and $\Delta^{*}=e v_{k_{1}+1,1}^{*}(P D[\Delta])$ as in (2.3). As in (2.1), the family GW invariants with constraint $P D(\sigma)$

$$
G W_{S+d F, g}^{\mathcal{H}}\left(P D(\sigma) ; \gamma_{1}, \cdots, \gamma_{k}\right)
$$

are obtained by evaluating (8.1) on the product of the classes $\gamma_{1}, \cdots, \gamma_{k}$.

The linearity of the parameter space $\mathcal{H}$ allows us to deform the spaces representing the summands of (8.1). Set $\Delta_{t}^{\mathcal{H}}=\{(\alpha, t \alpha) \mid \alpha \in \mathcal{H}\}$ and consider the spaces

$$
p_{1, d_{1}, d_{2}}^{-1}\left(\Delta_{t}^{\mathcal{H}}\right) \cap e v_{k_{1}+1,1}^{-1}(\Delta)
$$

for $0 \leq t \leq 1$. These consist of triples $\left(f_{1}, f_{2}, \alpha\right)$ where $f_{1}$ is $J_{\alpha}$-holomorphic and $f_{2}$ is $J_{t \alpha}$-holomorphic for the same $\alpha$. By Theorem $7.1 \mathrm{~b}$ the family moduli space for the class $S+d_{1} F$ is compact. That may not be true for the class $d_{2} F$, but nevertheless the spaces (8.2) are compact: the compactness of the space of $\left(f_{1}, \alpha\right)$ gives a bound on $|\alpha|$, which then implies the compactness of set of $\left(f_{2}, t \alpha\right)$. Consequently, as $t$ varies the spaces (8.2) trace out a compact cobordism, so represent the same 
homology class. Thus we can replace the summands of (8.1) by the classes of (8.2) with $t=0$, namely

$$
\left[\overline{\mathcal{M}}_{g_{1}, k_{1}+1}^{\mathcal{H}}\left(E(n), S+d_{1} F\right)\right] \otimes\left[\overline{\mathcal{M}}_{g_{2}, k_{2}+1}\left(E(n), d_{2} F\right)\right] \cap \Delta^{*}
$$

where the second factor is the ordinary GW moduli space. The same reasoning applies with $p_{1}$ replaced by $p_{2}$, so (8.1) becomes

$$
\begin{aligned}
& \sum_{i} \sum_{d=d_{1}+d_{2}}\left(\left[p_{i, d_{1}, d_{2}}^{-1}\left(\Delta^{\mathcal{H}}\right)\right] \cap \Delta^{*}\right) \\
& =\sum_{d=d_{1}+d_{2}}\left(\left[\overline{\mathcal{M}}_{g_{1}, k_{1}+1}^{\mathcal{H}}\left(E(n), S+d_{1} F\right)\right] \otimes\left[\overline{\mathcal{M}}_{g_{2}, k_{2}+1}\left(E(n), d_{2} F\right)\right] \cap \Delta^{*}\right) \\
& +\sum_{d=d_{1}+d_{2}}\left(\left[\overline{\mathcal{M}}_{g_{1}, k_{1}+1}\left(E(n), d_{1} F\right)\right] \otimes\left[\overline{\mathcal{M}}_{g_{2}, k_{2}+1}^{\mathcal{H}}\left(E(n), S+d_{2} F\right)\right] \cap \Delta^{*}\right) .
\end{aligned}
$$

We can then split the diagonal as in (2.4) to obtain a splitting formula for our family GW invariants.

Proposition 8.1 Let $\sigma$ be the gluing map of Figure 1 and fix cohomology bases $\left\{H_{a}\right\}$ and $\left\{H^{a}\right\}$ as in $(2.4)$. Then for any $\gamma_{1}, \cdots, \gamma_{k} \in H^{*}(E(n) ; \mathbb{Q})$

$$
\begin{aligned}
& G W_{S+d F, g}^{\mathcal{H}}\left(P D(\sigma) ; \gamma_{1}, \cdots, \gamma_{k}\right) \\
& =\sum_{a} \sum_{d=d_{1}+d_{2}} G W_{S+d_{1} F, g_{1}}^{\mathcal{H}}\left(\gamma_{1}, \cdots, \gamma_{k_{1}}, H_{a}\right) G W_{d_{2} F, g_{2}}\left(H^{a}, \gamma_{k_{1}+1}, \cdots, \gamma_{k}\right) \\
& +\sum_{a} \sum_{d=d_{1}+d_{2}} G W_{d_{1} F, g_{1}}\left(\gamma_{1}, \cdots, \gamma_{k_{1}}, H_{a}\right) G W_{S+d_{2} F, g_{2}}^{\mathcal{H}}\left(H^{a}, \gamma_{k_{1}+1}, \cdots, \gamma_{k}\right)
\end{aligned}
$$

where $G W$ denotes the ordinary GW invariants of $E(n)$.

Note that in this formula we multiply family GW invariants by ordinary GW invariants. Thus Proposition 8.1 can be used to "bootstrap" information about ordinary GW invariants in obtain information about family invariants.

The analogous formula for a non-separating node (as in Figure 2) especially straightforward. We have the exactly same second splitting formula as for GW invariant:

$$
\begin{aligned}
G W_{S+d F, g}^{\mathcal{H}}\left(P D(\theta) ; \gamma_{1}, \cdots, \gamma_{k}\right) & =\sum_{a} G W_{S+d F, g-1}^{\mathcal{H}}\left(\gamma_{1}, \cdots, \gamma_{k}, H_{a}, H^{a}\right) \\
& =(S+d F)^{2} G W_{S+d F, g-1}^{\mathcal{H}}\left(\gamma_{1}, \cdots, \gamma_{k}\right)
\end{aligned}
$$

where the second equality follows by the Divisor Axiom (1.7).

\section{Family Invariants for $E(n)$}

In this section, we will compute family invariants of $E(n)$ using the approach we took in Section 5 for $E(1)$ - combining TRR and symplectic sum formulas. We have just derived the relevant TRR formulas for the family invariants, so the next step is to similarly extend the Symplectic Sum Formula. Here we will do that for the relevant case of elliptic surfaces. 
The relevant sum formula is easily stated. Fix a smooth fiber $V$ of an elliptic fibration $E(n) \rightarrow S^{2}$ and, as before, regard $E(n)$ as the symplectic sum $E(n) \#_{V} E(0)$. Choose a smooth bump function $\beta$ that vanishes in a small $\delta$-neighborhood of $V$ and is 1 everywhere outside a $2 \delta$-neighborhood of $V$. We can define a set of relative family GW invariants for $(E(n), V)$ by the simple method of replacing $\alpha \in \mathcal{H}$ by $\beta \alpha$ in (7.1). Then each $J_{\beta \alpha}$ satisfies $J_{\beta \alpha}=J$ in a neighborhood of $V$. Using simple properties of $J_{\alpha}$-holomorphic maps, one can show that the resulting relative family moduli space for the classes $S+d F$ with $d \in \mathbb{Z}$ is compact. It then follows from the Symplectic Sum Theorem in Section 4 and the exactly same arguments of Section 5 that

$$
G W_{S+d F, 1}^{\mathcal{H}}(\tau(F))=\sum_{d_{1}+d_{2}=d} G W_{S+d_{1} F, 0}^{\mathcal{H}} \cdot G W_{S+d_{2} F, 1}(\tau(F)) .
$$

In fact, the proof of the sum formula given in [IP3] holds, essentially without change, for the family invariants provided that all the maps that arise in the limit $Z_{\lambda} \rightarrow Z_{0}$ lie in compact relative moduli spaces. That is true for a fibration $\lambda: \mathcal{Z} \rightarrow D$ whose generic fiber is $E(n)$ and whose center fiber is $E(n) \cup_{V} E(0)$ because the relative family moduli space is compact.

The splitting formulas in Section 8 and the sum formula (9.1) allow us to use the arguments of Section 5 to compute the genus 0 family GW invariants $G W_{S+d F, 0}^{\mathcal{H}}$ of $E(n)$ for the class $S+d F$. For these invariants, the family moduli space (with no marked points) has dimension $2(1-n)+2 p_{g}=0$. As before, we assemble the invariants in the generating function

$$
F(t)=\sum_{d \geq 0} G W_{S+d F, 0}^{\mathcal{H}} t^{d}
$$

and compare it with the generating function for the genus $g=1$ family invariants with the descendent constraint $\tau(F)$

$$
H(t)=\sum_{d \geq 0} G W_{S+d F, 1}^{\mathcal{H}}(\tau(F)) t^{d} .
$$

Applying the TRR formula as in (5.1) and (5.2) and simplifying using the splitting formulas in Section 8 gives

$$
\begin{aligned}
H(t) & =\frac{1}{12} \cdot \frac{1}{2} \sum_{d \geq 0}(S+d F)^{2} G W_{S+d F, 0}^{\mathcal{H}}(F) t^{d} \\
& +\sum_{d \geq 0}\left(\sum_{d_{1}+d_{2}=d} \sum_{a} G W_{S+d_{1} F, 0}^{\mathcal{H}}\left(F, H_{a}\right) \cdot G W_{d_{2} F, 1}\left(H^{a}\right)\right) t^{d} .
\end{aligned}
$$

As in (5.2)-(5.6), this simplifies after noting that (i) $(S+d F)^{2}=-n+2 d$, (ii) the canonical class $K$ of $E(n)$ is $(n-2) F$, and (iii) $d G W_{d F, 1}=(2-n) \sigma(d)$. Thus we obtain

$$
H(t)=\frac{1}{12} t F^{\prime}(t)-\frac{1}{12} F(t)+(2-n) G(t) F(t) .
$$

On the other hand, by the same arguments for (5.8) and the sum formula (9.1) yields

$$
H(t)=-\frac{1}{12} F(t)+2 G(t) F(t)
$$


Equating (9.2) and (9.3) gives the ODE

$$
\frac{1}{12} t F^{\prime}(t)=n G(t) F(t)
$$

with the initial condition $F(0)=G W_{S, 0}^{\mathcal{H}}=1$. The solution is the beautiful formula

$$
F(t)=\prod_{d \geq 1}\left(\frac{1}{1-t^{d}}\right)^{12 n}
$$

which generalizes the (5.9) for $E(1)$.

Another application of the sum formula gives the corresponding generating function for higher genus curves in $E(n)$; see [L2] for details. Bryan and Leung obtain exactly the same formulas by using a different set of family GW invariants of $E(n)$ (this extends the $E(1)$ and $E(2)$ cases done in [BL1]).

The exponent $12 n$ in (9.5) is the Euler characteristic of $E(n)$. Consequently, (9.5) is exactly the conjectured generating function of the rational curves in an $E(n)$ surface (see[Gö]). However, we do not know whether this count is enumerative, that is, whether it it the same as an actual count of holomorphic curves in a generic Kähler $E(n)$.

This issue arises because we have moved to a generic almost complex structure $J$, where we can apply symplectic cut-and-paste methods to find counts of curves. We can return to the Kähler case by taking a limit of generic $J$ that approach a Kähler $J_{0}$. Under that limit holomorphic curves converge to holomorphic curves, but degeneracies may appear: the limit curves might be multiply covered, and $J_{0}$ may admit families of curves when only a discrete collection of curves is expected. When such degeneracies are present it is not all clear when is meant by an enumeration of the curves. But this is an issue of algebraic geometry, quite separate from the symplectic invariant represented by the count of equation (9.5).

\section{The Yau-Zaslow Conjecture}

About a decade ago Yau and Zaslow [YZ] conjectured a formula counting rational curves in K3 surfaces. Let $N_{d}$ be the number of rational curves (i.e. curves whose domain has geometric genus 0) in K3 surfaces that represent some homology class $A$ with $A^{2}=2 d-2$. The conjecture is that the generating function for $N_{d}$ is

$$
\sum_{d \geq 0} N_{d} t^{d}=\prod_{d \geq 1}\left(\frac{1}{1-t^{d}}\right)^{24} .
$$

This is exactly the formula obtained in the previous section for curves in $E(2)=K 3$ in the classes $S+d F$. The Yau-Zaslow Conjecture asserts that the same formula counts all rational curves in a generic algebraic K3 surface.

Bryan and Leung [BL1] used their "twistor family invariants" to prove (10.1) for the cases where $A$ is a primitive class. An independent proof, also for primitive classes, was given by one of us (J. Lee) using symplectic methods ([L2]). In fact, we have just described that proof: equations (7.5) and (9.5) directly imply (10.1) for primitive classes in $E(2)=K 3$. The key point is that for primitive classes the count of holomorphic maps, given by the family GW invariants, is the same as the count of holomorphic curves, which are counted by the numbers $N_{d}$. 
Verifying the Yau-Zaslow Conjecture for multiple classes has turned out to be much harder. Any approach must deal with the fact that the counts of curves and maps are no longer the same because maps can multiply cover their images. Using algebraic geometry, A. Gathmann [Ga] worked out some special cases by hand, and Jun $\mathrm{Li}[\mathrm{Li}]$ outlined an approach. Very recently, the first author and N.C. Leung have extended the approach of the previous section to prove the Yau-Zaslow formula (10.1) for classes that are twice a primitive class. We will explain that result in this section. The key theorem is the following.

Theorem 10.1 ([LL]) Suppose that $X$ is a K3 surface and $A \in H_{2}(X ; \mathbb{Z})$ is twice a primitive class. Then the genus $g=0$ family $G W$-invariant of $X$ for the class $A$ is given by

$$
G W_{A, 0}^{\mathcal{H}}=G W_{B, 0}^{\mathcal{H}}+\left(\frac{1}{2}\right)^{3} G W_{A / 2,0}^{\mathcal{H}}
$$

where $B$ is any primitive class with $B^{2}=A^{2}$.

This theorem implies the Yau-Zaslow formula (10.1) for the classes that are twice a primitive class by the following reasoning. First, since we already know the Yau-Zaslow conjecture for primitive classes, the invariant $G W_{B, 0}^{\mathcal{H}}$ equals to $N_{d^{\prime}}$ where $d^{\prime}$ is defined by $B^{2}=A^{2}=2 d^{\prime}-2$. Similarly, since $A / 2$ is a primitive class, the number of curves representing $A / 2$ is $G W_{A / 2,0}^{\mathcal{H}}=N_{d}$ where $4(2 d-2)=2 d^{\prime}-2$. As explained by Gathmann [Ga], the double covers of those $A / 2$-curves contribute

$$
\left(\frac{1}{2}\right)^{3} G W_{A / 2,0}^{\mathcal{H}}
$$

to the invariant $G W_{A, 0}^{\mathcal{H}}$. Thus (10.2) is equivalent to the fact that the count of (reduced) rational curves representing the classes $A$ and $B$ are the same - both are the number given by formula (10.1).

Proof The approach is the same as above: relate the $g=1$ TRR formula and the symplectic sum formula for $E(2)=K 3$. This is done in detail in [LL]. Here is an outline.

First note that by (7.5) we can assume that $A / 2$ has the form $S+d F$ and $B=S+(4 d-3) F$; then $A^{2}=B^{2}=8(d-1)$. Thus it suffices to show that

$$
G W_{2(S+d F), 0}^{\mathcal{H}}=G W_{S+(4 d-3) F, 0}^{\mathcal{H}}+\left(\frac{1}{2}\right)^{3} G W_{S+d F, 0}^{\mathcal{H}} .
$$

This follows from

$$
\sum G W_{2 S+d F, 0}^{\mathcal{H}} t^{d}-\sum G W_{S+(2 d-3) F, 0}^{\mathcal{H}} t^{d}=\left(\frac{1}{2}\right)^{3} \sum G W_{S+d F, 0}^{\mathcal{H}} t^{2 d} .
$$

Note that if $d$ is odd both classes $2 S+d F$ and $S+(2 d-3) F$ are primitive with the same square and hence by (7.5) all odd terms in the left hand side of (10.3) vanish. Thus is suffices to show (10.3). That requires several steps.

We first derive a relation among the family invariants for the classes $2 S+d F$, $d \geq 0$. Fix a smooth fiber $V$ of $E(2) \rightarrow S^{2}$ and let

$$
G W_{2 S+d F, 1,(2)}^{V}\left(C_{F}\right)
$$


denote the genus 1 relative family invariants of $(E(2), V)$ and with multiplicity vector (2). This is a count of stable $J_{\alpha}$-holomorphic maps $f$ from an elliptic curve with one marked point $x$ so that $f$ contacts $V$ at $f(x)$ with multiplicity two and whose image has no components in $V$. Introduce generating functions

$$
M_{g}(\cdot)=\sum G W_{2 S+d F, g}^{\mathcal{H}}(\cdot) t^{d} \quad \text { and } \quad M_{1,(2)}^{V}=\sum G W_{2 S+d F, 1,(2)}^{V}\left(C_{F}\right) t^{d} .
$$

The genus 1 TRR formula combined with splitting formulas gives

$$
3 M_{1}(\tau(F))=t M_{0}^{\prime}-2 M_{0} .
$$

while the sum formula for the symplectic sum $E(2)=E(2) \#_{V} E(0)$ yields

$$
M_{1}(\tau(F))=M_{1,(2)}^{V}+4 G_{2} M_{0}
$$

and

$$
M_{2}(\tau(F), \tau(F))-2 M_{1}(p t)=20 G_{2} M_{1,(2)}^{V}+\left(16 G_{2}^{2}+8 t G_{2}^{\prime}\right) M_{0} .
$$

where $G_{2}(t)$ is the Eisenstein series with weight 2, namely

$$
G_{2}(t)=-\frac{1}{24}+G(t)=-\frac{1}{24}+\sum_{d \geq 0} \sigma(d) t^{d}
$$

(The coefficients of (10.5) and (10.6) consist of relative invariants of $E(0)$ that can be computed by several applications of genus 0 and 1 TRR formulas and the sum formula for the fiber sum $E(0)=E(0) \# E(0))$. Together, equations (10.4), (10.5), and (10.6) give

$$
\begin{aligned}
3 M_{2}(\tau(F), \tau(F))- & 6 M_{1}(p t) \\
& =20 t G_{2} M_{0}^{\prime}-\left(192 G_{2}^{2}+40 G_{2}-24 t G_{2}^{\prime}\right) M_{0} .
\end{aligned}
$$

We can repeat this argument to get a relation of invariants for the primitive classes $S+(2 d-3) F$. Note that we can always find an embedded symplectic submanifold $U$ of $E(2)$ that represents $2 F$ : take a map from an elliptic curve that double covers a fiber and perturb slightly; the perturbed map will remain symplectic, and will be an embedding by the adjunction formula since $F \cdot F=0$. Fix such a $U$ and write

$$
G W_{S+(2 d-3) F, 1,(2)}^{U}\left(C_{2 F}\right)
$$

for the genus 1 relative invariants of $E(2)$ relative to $U$ and multiplicity vector (2). We can then build two more generating functions:

$$
P_{g}(\cdot)=\sum G W_{S+(2 d-3) F, 0}^{\mathcal{H}}(\cdot) t^{d}, \quad P_{1,(2)}^{U}=\sum G W_{S+(2 d-3) F, 1}^{U}\left(C_{2 F}\right) t^{d} .
$$

For these, the genus 1 TRR formula gives a formula like (10.4) and the sum formula for the symplectic sum $E(2)=E(2) \#_{U} E(0)$ gives formulas like (10.5) and (10.6); together those give

$$
\begin{aligned}
3 P_{2}(\tau(F), \tau(F))-6 & P_{1}(p t) \\
& =20 t G_{2} P_{0}^{\prime}-\left(192 G_{2}^{2}+40 G_{2}-24 t G_{2}^{\prime}\right) P_{0} .
\end{aligned}
$$

Note that (10.7) and (10.8) have the same coefficients; that is true because all coefficients in the TRR and the sum formulas depend only on the topological quantities $(2 S+d F)^{2}=(S+(2 d-3) F)^{2}, \quad(2 S+d F) \cdot F=(S+(2 d-3) F) \cdot 2 F, \quad F^{2}=(2 F)^{2}$. 
Hence (10.7) and (10.8) give

$$
\begin{aligned}
& 3\left[M_{2}(\tau(F), \tau(F))-P_{2}(\tau(2 F), \tau(2 F))\right]-6\left[M_{1}(p t)-P_{1}(p t)\right] \\
& =20 G_{2} t\left[M_{0}-P_{0}\right]^{\prime}-\left(192 G_{2}^{2}+40 G_{2}-24 t G_{2}^{\prime}\right)\left[M_{0}-P_{0}\right]
\end{aligned}
$$

Next observe that because $2 S+d F$ and $S+(2 d-3) F$ are primitive classes with the same square when $d$ is odd, it follows from (7.5) that the generating functions $M_{1}(p t)-P_{1}(p t)$ and $M_{0}-P_{0}$ have no odd terms. One can also show that $M_{2}(\tau(F), \tau(F))-P_{2}(\tau(2 F), \tau(2 F))$ has no odd terms (see [LL2]). Therefore, comparing odd terms in both sides of (10.9) gives the first order ODE

$$
0=20 G_{o} t\left(M_{0}-P_{0}\right)^{\prime}-\left(384 G_{e} G_{o}+40 G_{o}-24 t G_{o}^{\prime}\right)\left(M_{0}-P_{0}\right)
$$

where $G_{e}(t)$ (resp. $\left.G_{o}(t)\right)$ is the sum of all even (resp. odd) terms of $G_{2}(t)$. The initial condition of this ODE is given by the well-known multiple map contribution [Ga]

$$
\left(M_{0}-P_{0}\right)(0)=G W_{2 S, 0}^{\mathcal{H}}=\left(\frac{1}{2}\right)^{3}
$$

On the other hand, it follows from the differential equation (9.4) that

$$
t \frac{d}{d t} F\left(t^{2}\right)=2 t^{2} F^{\prime}\left(t^{2}\right)=48 G\left(t^{2}\right) F\left(t^{2}\right)=48 G_{2}\left(t^{2}\right) F\left(t^{2}\right)+2 F\left(t^{2}\right) .
$$

Using this, one can check that $F\left(t^{2}\right)$ satisfies the same ODE (10.10) if

$$
-4 t^{2} G_{2}^{\prime}\left(t^{2}\right)+32 G_{2}^{2}\left(t^{2}\right)-40 G_{2}(t) G_{2}\left(t^{2}\right)+8 G_{2}^{2}(t)-t G_{2}^{\prime}(t)=0 .
$$

Under the substitution $t=e^{2 \pi i z}$ the left hand side of (10.11) is a modular form of level 2 with weight 4 and the space of those modular forms is a 2 dimensional vector space with well-known bases $[\mathrm{K}]$. Thus, one can prove (10.11) by showing the first two terms in the expansion of the left hand side of (10.11) vanish. Using the initial condition $F(0)=G W_{S, 0}^{\mathcal{H}}=1$, we can thus conclude that

$$
M_{0}(t)-P_{0}(t)=\left(\frac{1}{2}\right)^{3} F\left(t^{2}\right) .
$$

By the definition of $M_{0}(t)$ and $P_{0}(t)$, this is exactly the desired formula (10.3).

It would be interesting to extend this approach to higher multiple classes. Unfortunately that is not straightforward. In the above calculation, the right-hand side of the sum formula (10.6) fortuitously contains only genus 0 and 1 invariants of $E(2)$. That is a consequence of the vanishing of the genus 0 relative invariants of $E(0)$ constrained to contact three copies of $F$ to order 2. That vanishing does not occur for the classes $a S+d F$ with $a \geq 3$. That leads to additional terms in (10.6) for higher multiple classes, and dealing with those terms seems to require new ideas. 


\section{References}

[BL1] J. Bryan and N. C. Leung, The enumerative geometry of K3 surfaces and modular forms, J. Amer. Math. Soc. 13 (2000), 371-410.

[BL2] J. Bryan and N. C. Leung, Genenerating functions for the number of curves on abelian surfaces, Duke Math. J. 99 (1999), no. 2, 311-328.

[BL3] J. Bryan and N. C. Leung, Counting curves on irrational surfaces, Surveys in differential geometry: differential geometry inspired by string theory, 313-339, Surv. Diff. Geom., 5, Int. Press, Boston, MA, 1999.

$[\mathrm{BF}]$ K. Behrend and B. Fantechi, In Preparation.

[Ga] A. Gathmann, The number of plane conics 5-fold tangent to a given curve, preprint, math.AG/0202002.

[Ge] E. Getzler, Topological recursion relations in genus 2 , In "Integrable systems and algebraic geometry (Kobe/Kyoto, 1997)." World Sci. Publishing, River Edge, NJ, 198, pp 73-106.

[Gö] L. Göttsche, A conjectural generating function for numbers of curves on surfaces, Comm. Math. Phys. 196 (1998), no. 3, 523-533.

[IP1] E. Ionel and T. Parker, Gromov-Witten Invariants of Symplectic Sums, Math. Res. Lett., 5(1998), 563-576.

[IP2] E. Ionel and T. Parker, Relative Gromov-Witten Invariants, Annals of Math. 157 (2003), 45-96.

[IP3] E. Ionel and T. Parker, The Symplectic Sum Formula for Gromov-Witten Invariants, Annals of Math., 159 (2004), 935-1025.

[K] A.W. Knapp, Elliptic curves, Princeton University Press, 1992.

[L1] J. Lee, Family Gromov-Witten Invariants for Kähler Surfaces, Duke Math. J. 123 (2004), no $1,209-233$.

[L2] J. Lee, Counting Curves in Elliptic Surfaces by Symplectic Methods, preprint, math. SG/0307358.

[LL] J. Lee and N.C. Leung, Yau-Zaslow formula on K3 surfaces for non-primitive classes, preprint, math. SG/0404537.

[LL2] J. Lee and N.C. Leung, Counting elliptic curves in K3 surfaces, preprint, math. SG/0405041.

[Li] J. Li, A note on enumerating rational curves in a K3 surface, Geometry and nonlinear partial differential equations (Hangzhou, 2001), 53-62, AMS/IP Stud. Adv. Math. 29, Amer. Math. Soc., Providence, RI, 2002.

[LT] J. Li and G. Tian, Virtual moduli cycles and Gromov-Witten invariants of general symplectic manifolds, Topics in symplectic 4-manifolds (Irvine, CA, 1996), 47-83, First Int. Press Lect. Ser., I, International Press, Cambridge, MA, 1998.

[P] T. Parker, Compactified moduli spaces of pseudo-holomorphic curves Mirror symmetry, III (Montreal, PQ, 1995), 77-113, AMS/IP Stud. Adv. Math., 10, Amer. Math. Soc., Providence, RI, 1999.

[PW] T. Parker and J. Wolfson, Pseudo-holomorphic maps and bubble trees, Jour. Geometric Analysis, 3 (1993) 63-98.

[RT1] Y. Ruan and G. Tian, A mathematical theory of quantum cohomology, J. Differential Geom. 42 (1995), 259-367.

[RT2] Y. Ruan and G. Tian, Higher genus symplectic invariants and sigma models coupled with gravity, Invent. Math. 130 (1997), 455-516.

[IS] S. Ivashkovich and V. Shevchishin, Gromov compactness theorem for J-complex curves with boundary, Internat. Math. Res. Notices 22 (2000), 1167-1206.

[YZ] S.T. Yau and E. Zaslow, BPS States, String Duality, and Nodal Curves on K3, Nuclear Phys. B 471 (1996), 503-512. 